\title{
El liderazgo en relaciones públicas y gestión de comunicación. Análisis cuantitativo de los factores de liderazgo en el sector en España
}

\section{María de los Ángeles Moreno' \\ Cristina Navarro ${ }^{2}$ María Luisa Humanes ${ }^{3}$}

Recibido: 2013-06-23

Enviado a pares: 2013-06-25
Aprobado por pares: 2013-07-10

Aceptado: 2014-02-14

DOI: 10.5294/pacla.2014.17.3.16

Para citar este artículo / To reference this article / Para citar este artigo Moreno, M. A., Navarro, C., Humanes M.L. Septiembre 2014. El liderazgo en relaciones pùblicas y gestión de comunicación. Análisis cuantitativo de los factores de liderazgo en España. Palabra Clave 17 (3), 946-978. D0I: 10.5294/pacla.2014.17.3.16

\section{Resumen}

Este artículo analiza el liderazgo en el sector de las relaciones públicas y la gestión de la comunicación en España. Siguiendo el modelo conceptual de Meng y Berger (2011), se abordan los requisitos y características de los 'líderes excelentes', sus creencias y percepciones sobre el liderazgo, los desafíos que afrontan en la actualidad, así como las estrategias y acciones que implementan para superar con éxito los retos que se presentan en el desempeño de su trabajo. Esta investigación forma parte de un proyecto internacional desarrollado en 23 países, promovido por el Plank Center for Leadership in Public Relations de la Universidad de Alabama y financiado por IBM y Heyman Associates en Estados Unidos.

\footnotetext{
Universidad Rey Juan Carlos, España. mariaangeles.moreno@urjc.es.

Universidad Rey Juan Carlos, España. cristina.navarro@urjc.es.

Universidad Rey Juan Carlos, España marialuisa.humanes@urjc.es.
} 


\section{Palabras clave}

Liderazgo, Relaciones Públicas, Gestión de la comunicación, Percepciones, Estrategias (Fuente: Tesauro de la Unesco).

\section{Leadership in Public Relations and Communication Management. A Quantitative Analysis of Leadership Factors in the Industry in Spain}

\section{Abstract}

Leadership in public relations and communication management in Spain is analyzed in this paper. Following Meng and Berger's conceptual model (2011), the prerequisites and characteristics of outstanding leaders in communication are examined, as are their personal beliefs and perceptions about leadership, the major challenges facing Spanish leaders in public relations and communication management, and the strategies implemented to deal with those issues. This study is part of an international project developed in 23 countries by the Plank Center for Leadership in Public Relations at the University of Alabama. It was financed by Heyman Associates in New York and the IBM Corporation.

\section{Keywords}

Leadership, public relations, communication management, perceptions, strategies (Source: UNESCO Thesaurus). 


\section{A liderança em relações públicas e gestão de comunicação. Análise quantitativa dos fatores de liderança no setor na Espanha}

\section{Resumo}

Este artigo analisa a liderança no setor das relações públicas e a gestão da comunicação na Espanha. Seguindo o modelo conceitual de Meng e Berger (2011), abordam-se os requisitos e características dos "líderes excelentes”, suas crenças e percepções sobre a liderança, os desafios que enfrentam na atualidade, assim como as estratégias e ações que implementam para superar com sucesso os desafios que se apresentam no desempenho de seu trabalho. Esta pesquisa faz parte de um projeto internacional desenvolvido em 23 países, promovido pelo Plank Center for Leadership in Public Relations da Universidade do Alabama e financiado pela IBM e Heyman Associates nos Estados Unidos.

\section{Palavras-chave}

Liderança, relações públicas, gestão da comunicação, percepções, estratégias (Fonte: Tesauro da Unesco). 


\section{Introducción y estado de la cuestión}

El liderazgo en relaciones públicas y comunicación estratégica ha sido ampliamente estudiado desde diferentes ángulos y enfoques, centrándose de forma específica en la importancia de desarrollar habilidades y aplicar estilos de liderazgo apropiados para lograr una comunicación efectiva. Sin embargo, tan sólo una pequeña parte de los artículos publicados hasta el momento han sugerido que los líderes en relaciones públicas desempeñan un papel importante para el éxito, la imagen y el futuro de las organizaciones (Aldoory y Toth; Berger y Meng; Berger y Reber, 2006; Berger, Reber y Heyman, 2007; Grunig, Grunig y Dozier, 2002; Werder y Holtzhausen, 2009). Los líderes del sector de la gestión de la comunicación influyen en el comportamiento, las actitudes y creencias de los empleados y determinan tanto la cultura corporativa como el clima de comunicación. Además, el trabajo que desempeñan permite incrementar el valor de las relaciones públicas, aumentar la eficacia de la organización y facilitar la toma de decisiones correctas (Berger y Meng, 2010).

Uno de los principales obstáculos a la hora de abordar el tema del liderazgo reside en la complejidad del término, la diversidad de definiciones planteadas -muchas veces contradictorias entre sí- y la falta de consenso sobre el alcance real de la influencia de los líderes sobre el grupo y el comportamiento de la organización. Se trata de un fenómeno complejo, que obliga a aproximarse a él desde diferentes niveles, incluyendo los rasgos psicológicos individuales, las formas de interacción con el grupo, la cultura y los valores organizacionales o los modelos de rol (Meng y Berger, 2011).

A lo largo del último siglo, las investigaciones en este campo han ido evolucionando, desde una concepción basada en los rasgos del líder a otras más sofisticadas centradas en el análisis de estilos y comportamientos, habilidades técnicas y humanas, trabajo y dirección de grupos, enfoques situacionales, modelo de contingencia, hasta llegar al denominado liderazgo auténtico (Northouse, 2007). Todas estas aproximaciones han dado origen a un extenso corpus de conocimiento en el que las teorías del liderazgo transformacional (Bass, 1985; House, 1971; 1977; Bass y Avolio, 1993, 
1995), con su combinación de dimensiones racionales y afectivas, han sido las más aceptadas y difundidas.

\section{El liderazgo en relaciones públicas y gestión de la comunicación}

A pesar de que las organizaciones son cada vez más conscientes de la importancia estratégica de la comunicación para poder competir y alcanzar sus objetivos en un mundo globalizado, pocos estudios han abordado directamente las características propias del liderazgo en relaciones públicas y han tratado de conceptualizar las dimensiones que determinan su efectividad.

Sin embargo, pese a la escasez de investigaciones específicas sobre esta materia, la noción de liderazgo está implícita en varias aproximaciones teóricas clásicas del área de las relaciones públicas y, de hecho, algunos estudios en el campo de la comunicación han subrayado la importancia de aplicar destrezas o habilidades relacionadas con el liderazgo para mejorar el trabajo de los profesionales y ayudarles a participar con éxito en la toma estratégica de decisiones (Berger y Reber, 2006; Berger et al., 2007; Gruning et al., 2002; Smudde y Courtright, 2010; Werder y Holtzhausen, 2009).

En este sentido, los principios de la ampliamente aceptada teoría de la excelencia en relaciones públicas (Broom y Dozier, 1986; Dozier y Broom, 1995; Grunig, 1992) señalan que los líderes en este campo deben: a) estar implicados en la gestión estratégica de la organización; b) formar parte de la coalición dominante; c) poseer un punto de vista gerencial, conocimiento y experiencia profesional, y d) utilizar la comunicación bidireccional. Los autores de la teoría de la excelencia también afirman que la estructura y la cultura de la organización influyen en la efectividad del trabajo del profesional de las relaciones públicas y abogan por una 'cultura para la comunicación' en la que se favorezca la participación, la simetría y la igualdad de oportunidades.

Por su parte, la teoría de contingencia de relaciones públicas (Cameron, Cropp y Reber, 2001; Reber y Cameron, 2003), centrada en las relaciones estratégicas y conflictivas entre una organización y sus públicos, 
sugiere que los líderes deben ser capaces de analizar y evaluar las amenazas y oportunidades externas, elegir la posición adecuada en cada momento y promover de forma efectiva las relaciones con el resto de los líderes de la organización. En contraste con las teorías 'universales', y con la normatividad de la teoría de la excelencia, los teóricos de la contingencia discrepan de la existencia de un único modelo de liderazgo excelente y su investigación está encaminada a identificar variables situacionales que puedan proponer diferentes estilos de liderazgo (Horner-Long y Schoenberg, 2002).

Desde la perspectiva de la teoría organizacional, algunos estudios han aproximado los estilos de liderazgo al ámbito de la comunicación estratégica. Aldoory y Toth (2004) han investigado los estilos más efectivos para el sector de las relaciones públicas y las diferentes percepciones en función del género, descubriendo la preferencia de los profesionales por el estilo transformacional y la mayor dificultad de las mujeres para acceder a las posiciones de líder. Werder y Holzhausen (2009), en esta misma línea, desarrollaron una investigación empírica que permitió detectar tres modelos aplicables al ámbito de las relaciones públicas: el transaccional, que se basa en la autoridad; el transformacional, que apela a ideales y visiones compartidas, $y$ el inclusivo, que detecta los retos y estimula la toma de decisiones conjuntas. Estos autores subrayan la prevalencia de los estilos transformacional e inclusivo en las relaciones con el entorno y afirman que ambos contribuyen a incrementar la eficacia de las estrategias de relaciones públicas. En el contexto europeo, Zerfass, Verhoeven, Tench, Moreno y Vercic (2011) afirman que los profesionales de la gestión de la comunicación que acceden a los niveles más altos dentro del organigrama y aplican un estilo de liderazgo inclusivo obtienen mayores cuotas de poder. Estos investigadores también concluyen que el estilo aparece determinado por el tipo de organización, por la cultura organizacional (interactiva, integrada, sistematizada y emprendedora) y por la capacidad de participar en la toma de decisiones.

Otros investigadores también han profundizado en los factores ligados al éxito y al liderazgo en la profesión. En esta línea, Berger, Reber y Heyman (2007) señalan algunos elementos y patrones que consideran cruciales 
para el líder, incluyendo habilidades de comunicación y persuasión, capacidad de comunicación interpersonal, una naturaleza positiva y proactiva, además de una experiencia laboral variada. Choi y Choi (2009) desarrollan un enfoque conductista e identifican seis comportamientos de liderazgo que podrían condicionar el valor de las relaciones públicas, incluyendo la necesidad de incorporar al organigrama a personas que posean una visión clara de las estrategias de relaciones públicas, ejerciendo una sólida influencia, actuando como agentes de cambio y creando alianzas dentro y fuera de la organización.

Tras realizar un minucioso análisis de la literatura científica previa, Meng y Berger (2010) proponen una definición universal de liderazgo excelente que trata de sintetizar el amplio abanico de perspectivas sobre este complejo y multifacético fenómeno:

\begin{abstract}
El liderazgo excelente en relaciones públicas es un proceso dinámico que abarca un conjunto de habilidades individuales y atributos personales, valores y comportamientos que producen comunicación efectiva y ética de forma consistente. Esta práctica alimenta y guía el éxito de los equipos de comunicación, ayuda a las organizaciones a lograr sus metas y las legitima socialmente (p. 427).
\end{abstract}

Además, Meng y Berger (2011) señalan que el liderazgo en relaciones públicas es una compleja mezcla de seis dimensiones interrelacionadas: 1) dinamismo personal (autoconocimiento y visión compartida), 2) trabajo en equipo, 3 ) orientación ética, 4) construcción de relaciones (externas e internas), 5) toma de decisiones estratégicas y 6) comunicación y gestión del conocimiento. Este modelo conceptual de carácter integrador entiende que los profesionales pueden utilizar rasgos específicos y conocimientos para reducir las limitaciones que encuentran, reforzar las relaciones internas y externas e influir de forma favorable en la toma de decisiones. Además, sostiene que los atributos asociados con una cultura específica predicen la estructura organizacional e influyen en el comportamiento de las relaciones públicas y que la consecución de un liderazgo excelente puede estar condicionada por influencias situacionales (Meng, Berger, Gower y Heyman, 2012). 
Basándose en este modelo y en el metaanálisis de 16 estudios sobre liderazgo, Berger y Meng (2010) proponen nueve principios para lograr la excelencia en este campo y que, en su conjunto, representan un enfoque normativo para el desarrollo de una teoría del liderazgo en comunicación estratégica. Estos autores concluyen que los líderes excelentes en relaciones públicas deberían poseer las siguientes cualidades: 1) liderar con el ejemplo; 2) participar de forma efectiva y creíble en la toma de decisiones estratégicas dentro de la organización; 3 ) ejemplificar una fuerte orientación ética; 4) tener complejas habilidades comunicativas y retóricas; 5) disponer de un profundo autoconocimiento que les permita guiar con éxito todo tipo de interacciones, formación y relaciones, además del desarrollo personal; 6) disponer de un fuerte deseo de liderar; 7) utilizar estilos de liderazgo transformacionales e inclusivos, sensibles al medio ambiente y al entorno, además de a las diferencias y las necesidades individuales; 8) demostrar pasión por el trabajo y la profesión, animando e inspirando a los demás, y 9) actuar como agentes de cambio, ayudando a crear una cultura de comunicación.

\section{Investigación sobre liderazgo en relaciones púbicas en España}

En España, las investigaciones publicadas hasta el momento ofrecen una tímida radiografía sobre aspectos relacionados con la posición del líder de comunicación dentro del organigrama de la empresa, su participación en los niveles directivos, la posibilidad de rendir cuentas ante el primer ejecutivo (chief executive officer), las responsabilidades que asume, su capacidad de influencia y el estilo de liderazgo que ejerce (Costa, 2009, 2011; Fernández y Huertas, 2005; Gutiérrez-García, 2010; Matilla, 2010; Matilla y Marca, 2011; Morales y Enrique, 2007; Moreno, 2004; Moreno, Zerfass y Navarro, 2010, 2011, 2012; Mut, 2006). Sin embargo, hasta el momento no se ha profundizado en las habilidades, actitudes o comportamientos que favorecen un liderazgo excelente, ni en las percepciones o creencias del líder respecto al trabajo que desempeña.

Uno de los estudios que ha arrojado datos más interesantes sobre este campo es el European Communication Monitor (ECM), una iniciativa de 
Euprera (European Public Relations Education and Research Association), que en 2007 comenzó a identificar los retos actuales y desarrollos futuros de las relaciones públicas en Europa. Según datos del ECM 2011 relativos a España, el 44\% de los líderes inclusivos tiene peso en las decisiones gerenciales, por tan sólo el 33\% de los transaccionales (Moreno, Zerfass y Navarro, 2012). Hay que tener en cuenta, además, que entre los profesionales españoles sólo el $27,5 \%$ se ocupa de todos los ámbitos de comunicación y stakeholders dentro de su organización, frente al $41,7 \%$ de los europeos. La mayoría de los españoles (55\%) es responsable de al menos tres campos de comunicación y grupos de stakeholders, mientras que el trabajo del 10\% se limita exclusivamente a las relaciones con los medios de comunicación y a la comunicación externa.

Este estudio también señala que en España los profesionales consideran muy relevante el papel que desempeñan dentro de la organización. En este sentido, el $87 \%$ cree que el departamento se anticipa a las situaciones y temas en conflicto, el $82 \%$ señala que juega un papel importante en el rendimiento global de la organización e incluso el $80 \%$ asegura que 'es insustituible', afirmación que suscribe el $69,8 \%$ de los profesionales del resto de Europa.

La posición del profesional de relaciones públicas en España es muy similar a la del resto de Europa y en ambos casos el $60 \%$ rinde cuentas directamente al primer ejecutivo de la compañía y un $17 \%$ a otro miembro del consejo de administración. Por otra parte, el 22,5\% de los líderes españoles en relaciones públicas forma parte del consejo de administración, frente al 17,8\% de los europeos. De estos datos se desprende que en España la posición del departamento de comunicación o relaciones públicas es, en general, favorable e incluso goza de un peso mayor que en el resto del continente (Moreno et al., 2012).

La Asociación de Directivos de Comunicación (Dircom, 2000, 2005, $2008,2010,2011)$ y la Asociación de Consultores en Relaciones Públicas y Comunicación (Adecec, 2002, 2004, 2008), las dos organizaciones profesionales mayoritarias de ámbito nacional, también han hecho un esfuerzo 
por analizar la figura del líder de relaciones públicas en las empresas españolas. Según una investigación conducida por Dircom (2011), las habilidades más importantes para desempeñar con éxito el trabajo son: capacidad de comunicación, accesibilidad a los medios, influencia en la dirección de la empresa, empatía, alto conocimiento de los productos y servicios de la compañía, creatividad, autoridad y liderazgo. Respecto al estatus que ocupan los directores de comunicación en el organigrama empresarial, esta investigación confirma un incremento de la importancia de este cargo, que en el 75\% de los casos depende directamente del presidente, director general o consejero delegado. Entre las actividades asignadas, las más valoradas son los contactos con la alta dirección, las relaciones con los medios de comunicación, el diseño de estrategias, la relación con los públicos externos y las relaciones con otras áreas de la empresa, quedando en segundo plano la gestión financiera del departamento.

Por su parte, un estudio realizado por la Fundación EOI (2010) confirma que la mayoría de los directores de comunicación españoles $(53,4 \%)$ dependen jerárquicamente de los dos primeros niveles de la empresa y que cada vez es más habitual su presencia en los comités de dirección. Su puesto es definido como claramente estratégico dentro del funcionamiento de la organización, aunque su papel sigue estando excesivamente ligado a la comunicación externa y a las relaciones con los medios de comunicación.

\section{Metodología}

Este estudio se plantea tres objetivos principales: 1) identificar los retos de la profesión en España y su relación con las dimensiones de liderazgo, 2) apuntar las tendencias para el desarrollo de los líderes del futuro en el sector y 3) determinar las creencias personales y percepciones sobre el liderazgo de los profesionales del sector. En definitiva, se han formulado las siguientes preguntas de investigación:

PI1: ¿Cuáles son los retos o asuntos más importantes que influyen en la función de los líderes en relaciones públicas y gestión de la comunicación?

PI2. ¿Cómo afectan estos retos a las dimensiones del liderazgo?

PI3. ¿Qué estrategias o acciones ayudan a gestionar estos retos? 
PI4. ¿Cómo se puede mejorar el desarrollo de los líderes del futuro?

PI5. ¿Cuáles son las creencias personales y percepciones sobre el liderazgo?

Para alcanzar estos objetivos se ha llevado a cabo una encuesta online, distribuida a profesionales españoles de las relaciones públicas y la gestión de la comunicación, empleando una base de datos elaborada exclusivamente para la investigación. Los encuestados debían estar en activo y trabajando en organizaciones de diferente naturaleza (entidades públicas, empresas, agencias de comunicación, organizaciones sin ánimo de lucro, etc.), con distintos puestos de responsabilidad y años de experiencia.

Este estudio forma parte de un proyecto más amplio, liderado por el Plank Center for Leadership in Public Relations de la Universidad de Alabama (EUA), y financiado por IBM y Heyman Associates de New York. La investigación global, que ha empleado metodología cuantitativa y cualitativa, se ha llevado a cabo en ocho regiones culturales y 23 países: Brasil, Canadá, Chile, China, Hong Kong, Singapur, Taiwán, Estonia, Letonia, Egipto, Austria, Alemania, Suiza, India, Líbano, México, Arabia Saudita, Corea del Sur, España, Emiratos Árabes, Reino Unido, Rusia y Estados Unidos.

El cuestionario se elaboró tras una rigurosa revisión de la literatura científica existente, fue redactado en inglés y probado en dos fases: una inicial en la que participaron 12 expertos de diferentes países y otra final con 57 profesionales internacionales. El cuestionario, compuesto por 58 preguntas distribuidas en cuatro secciones, se tradujo y adaptó a nueve idiomas. Cada pregunta permitía respuestas múltiples y una gradación de siete valores en escalas de actitud. La primera sección aborda la percepción sobre asuntos clave y cambios en el entorno, además de aspectos organizacionales y estructurales; una segunda parte analiza las vías para mejorar el desarrollo de los futuros líderes y fortalecer la profesión; la tercera se centra en las creencias y percepciones sobre el liderazgo, mientras que la cuarta incluye preguntas demográficas. El estudio global obtuvo una participación de 4484 profesionales de gestión de comunicación en todo el mundo. 
En España la encuesta se distribuyó vía e-mail junto con una invitación personal durante los meses de enero y febrero de 2012, y el número final de cuestionarios completos respondidos fue de 208. Los participantes habían sido claramente identificados como profesionales de las relaciones públicas y la gestión de la comunicación en activo en España, con representación de todos los sectores objetivo: entidades públicas, empresas, agencias de comunicación, organizaciones sin ánimo de lucro y freelancers con distintos puestos de responsabilidad y años de experiencia.

Los datos demográficos indican que el 50,5\% de la muestra está compuesta por mujeres $(n=105)$ y el $49,5 \%$ por hombres $(n=103)$. La mayoría lleva trabajando en el campo de las relaciones púbicas entre 11 y 20 años $(n=96,46,2 \%)$, preferentemente en organizaciones gubernamentales, sin ánimo de lucro, políticas o del sector educativo $(\mathrm{n}=77,37 \%)$, agencias $(n=48,23,1 \%)$ y organizaciones privadas $(n=44,21,2 \%)$. Respecto a la edad, el grupo más numeroso es el integrado por personas de entre 36 y 45 años $(n=78,37,5 \%)$, en posesión de una licenciatura universitaria $(n=112,53,8 \%)$ en el campo de estudio del periodismo $(n=96,46,2 \%)$. Además, la mayor parte ocupa la posición de líder de la unidad o grupo de comunicación $(\mathrm{n}=78,37,5 \%)$ integrado por menos de cinco profesionales $(\mathrm{n}=115,55,3 \%)$.

Los datos fueron sometidos a análisis estadístico descriptivo y multivariado. En concreto se realizó un análisis factorial de componentes principales con los 12 ítems relacionados con las acciones necesarias para la mejora del liderazgo en los profesionales del futuro.

\section{Análisis y resultados}

\section{Los retos profesionales más importantes para el líder de comunicación}

Resulta evidente que la introducción de nuevos canales de comunicación, más interactivos y participativos que los tradicionales, ha removido los cimientos de las relaciones públicas y está obligando a los líderes del sector a hacer frente a problemas hasta ahora desconocidos. De hecho, la mayo- 


\section{Tabla 1}

\section{Perfil demográfico de los encuestados}

\begin{tabular}{|c|c|c|}
\hline \multirow{2}{*}{ Variables } & \multicolumn{2}{|c|}{ Total muestra tamaño $(\mathrm{N}=\mathbf{2 0 8})$} \\
\hline & Frecuencia $(\mathbf{n})$ & Porcentaje \\
\hline \multicolumn{3}{|l|}{ Género } \\
\hline Hombre & 103 & 49,5 \\
\hline Mujer & 105 & 50,5 \\
\hline \multicolumn{3}{|l|}{ Edad } \\
\hline Menos de 36 años & 52 & 25 \\
\hline $36-45$ años & 78 & 37,5 \\
\hline $46-55$ años & 53 & 25,5 \\
\hline Más de 55 años & 25 & 12 \\
\hline \multicolumn{3}{|c|}{ Años de experiencia profesional en relaciones públicas } \\
\hline Menos de 11 años & 69 & 33,2 \\
\hline Entre 11 y 20 años & 96 & 46,2 \\
\hline Más de 20 años & 43 & 20,7 \\
\hline \multicolumn{3}{|c|}{ Tipo de organización para la que trabaja } \\
\hline Entidad pública & 15 & 7,2 \\
\hline Entidad privada & 44 & 21,2 \\
\hline Organización sin ánimo de lucro, gubernamental, política o educativa & 77 & 37 \\
\hline Agencia & 48 & 23,1 \\
\hline Autónomo & 11 & 5,3 \\
\hline \multicolumn{3}{|c|}{ Niveles entre su posición y el líder de más alto rango en su organización } \\
\hline 0 (soy el líder principal en relaciones públicas) & 78 & 37,5 \\
\hline 1 nivel informativo & 70 & 33,7 \\
\hline Combinación 2, 3, $4 \mathrm{u}$ otras opciones & 59 & 28,4 \\
\hline \multicolumn{3}{|c|}{ Número de profesionales que integran la unidad o departamento de comunicación } \\
\hline Menos de 5 profesionales & 115 & 55,3 \\
\hline Entre 5 y 15 profesionales & 64 & 30,8 \\
\hline Entre 16 y 25 profesionales & 12 & 5,8 \\
\hline Más de 25 profesionales & 17 & 8,2 \\
\hline \multicolumn{3}{|l|}{ Nivel educativo } \\
\hline Bachillerato o preparatoria & 6 & 2,9 \\
\hline Licenciatura/Título profesional & 112 & 53,8 \\
\hline Máster o maestría & 66 & 31,7 \\
\hline Doctorado & 22 & 10,6 \\
\hline \multicolumn{3}{|l|}{ Área de estudio } \\
\hline Publicidad o marketing & 16 & 7,7 \\
\hline Administracion de empresas, economía o gestión & 15 & 7,2 \\
\hline
\end{tabular}




\begin{tabular}{|l|c|c|}
\hline \multicolumn{1}{|c|}{ Variables } & \multicolumn{2}{c|}{ Total muestra tamaño (N=208) } \\
\cline { 2 - 3 } & Frecuencia (n) & Porcentaje \\
\hline Informática (ciencias computacionales) & 1 & 0,5 \\
\hline Comunicación y estudio de medios & 10 & 4,8 \\
\hline Humanidades (historia, lingüística, literatura, filosofía, lenguaje, etc.) & 11 & 5,3 \\
\hline Periodismo & 96 & 46,2 \\
\hline Matemáticas o ciencias naturales (biología, química, física, etc.) & 3 & 1,4 \\
\hline $\begin{array}{l}\text { Relaciones públicas, gestión de la comunicación, comunicación estratégica, } \\
\text { comunicación corporativa, comunicación organizacional, o RR. PP. y publicidad }\end{array}$ & 25 & 12 \\
\hline ciencias sociales (antropología, estudios políticos, psicología, sociología, etc.) & 10 & 4,8 \\
\hline Otros & 17 & 8,2 \\
\hline
\end{tabular}

ría de los profesionales españoles, al ser preguntados sobre el reto más relevante en el trabajo diario del líder de comunicación, se decantan por la necesidad de gestionar la revolución digital y el crecimiento de los social media $(\mathrm{n}=45,21,6 \%)$, seguido muy de cerca por el desafío de abordar la rapidez y el gran volumen del flujo informativo $(n=43,20,7 \%)$. Estos datos demuestran que las organizaciones necesitan líderes capaces de identificar y cribar, entre la avalancha de información que reciben del entorno, lo importante de lo intrascendente, actuando casi como intérpretes y canalizadores de la información.

Mejorar la medición de la eficacia de la comunicación para demostrar su valor y estar preparados para afrontar con eficacia las posibles crisis $(n=26,12,5 \%)$ ocupan el tercer puesto conjuntamente. En los últimos puestos aparecen, como cuestiones menos importantes, la necesidad de mejorar la imagen de la profesión $(n=5,2,4 \%)$ y de hacer frente a la creciente demanda de responsabilidad social corporativa $(n=6,2,9 \%)$.

\section{Requisitos previos para que los líderes de relaciones públicas puedan afrontar con éxito los retos más importantes de la profesión}

Respecto a las condiciones, cualidades o habilidades necesarias para que los líderes puedan hacer frente a los retos que plantea el principal asunto de interés seleccionado previamente, los encuestados destacan la importancia de participar en la toma de decisiones estratégicas dentro de su organización $(M=6,22, S D=1,097)$. Esta condición concuerda con los principios 


\section{Tabla 2}

\begin{tabular}{|l|c|c|}
\hline \multicolumn{1}{|c|}{ Los asuntos más importantes para el líder de comunicación } & Frecuencia & Porcentaje \\
\hline La revolución digital y el crecimiento de los social media & 45 & 21,6 \\
\hline Lidiar con la velocidad y el volumen del flujo de información & 43 & 20,7 \\
\hline Mejorar la imagen de la profesión de relaciones públicas/gestión de comunicación & 26 & 12,5 \\
\hline Estar preparados para afrontar con eficacia las posibles crisis & 26 & 12,5 \\
\hline Afrontar la creciente demanda de transparencia en comunicaciones y operaciones & 24 & 11,5 \\
\hline Buscar, desarrollar y conservar a los profesionales de comunicación con gran talento & 12 & 5,8 \\
\hline Enfrentar las necesidades de comunicación en culturas diversas y mercados globalizados & 11 & 5,3 \\
\hline Mejorar el compromiso de los empleados y la responsabilidad en el trabajo & 10 & 4,8 \\
\hline Hacer frente a la creciente demanda de responsabilidad social corporativa & 6 & 2,9 \\
\hline Mejorar la imagen de la profesión de relaciones públicas/gestión de comunicación & 5 & 2,4 \\
\hline Total & 208 & 100 \\
\hline
\end{tabular}

generales postulados por la teoría de la excelencia (J. E. Grunig, 1992; L. A. Grunig et al., 2002), en la que se afirma que el líder en relaciones públicas debe estar implicado directamente en las decisiones de la organización, formando parte de la coalición dominante.

Le siguen, por orden de importancia, los siguientes requisitos: poseer conocimientos sobre comunicación para desarrollar estrategias, planes y mensajes apropiados $(M=6,17, S D=1,084)$, ofrecer una visión convincente de cómo la comunicación puede ayudar a la organización $(\mathrm{M}=6,02, \mathrm{SD}=1,097)$, tener habilidad para crear y gestionar equipos profesionales de trabajo $(M=5,94, S D=1,130)$, disponer de una fuerte orientación ética y un conjunto de valores que guíen las acciones $(M=5,84$, $\mathrm{SD}=1,243)$, trabajar en una organización que apoye la comunicación bidireccional y el poder compartido $(\mathrm{M}=5,81, \mathrm{SD}=1,355)$ y tener habilidad para desarrollar coaliciones internas y externas a la organización $(M=5,77$, $\mathrm{SD}=1,245)$.

\section{Estrategias y acciones implementadas por los equipos de comunicación}

Además de identificar claramente los principales retos para el sector y para los líderes en relaciones públicas, este estudio también tenía como objetivo conocer las principales estrategias desarrolladas a la hora de afrontar los desafíos previamente señalados. 


\section{Tabla 3}

\begin{tabular}{|l|c|c|}
\hline \multicolumn{1}{|c|}{ Evaluación de las condiciones más importantes, habilidades o cualidades de liderazgo } & M & SD \\
\hline Participar en la toma de decisiones estratégicas de su organización en relación con el asunto de interés & 6,22 & 1,097 \\
\hline Poseer conocimientos sobre comunicación para desarrollar estrategias, planes y mensajes apropiados & 6,17 & 1,084 \\
\hline Ofrecer una visión convincente de cómo la comunicación puede ayudar a la organización & 6,02 & 1,061 \\
\hline Tener habilidad para crear y gestionar equipos profesionales de trabajo para tratar el asunto de interés & 5,94 & 1,130 \\
\hline Disponer de una fuerte orientación ética y un conjunto de valores que guíen las acciones & 5,84 & 1,243 \\
\hline Trabajar en una organización que apoye la comunicación bidireccional y el poder compartido & 5,81 & 1,355 \\
\hline $\begin{array}{l}\text { Tener habilidad para desarrollar coaliciones internas y externas a la organización para gestionar el asunto } \\
\text { de interés }\end{array}$ & 5,77 & 1,245 \\
\hline
\end{tabular}

Nota: Escala Likert de 7 puntos entre 'poco importante' (1) y 'muy importante' (7).

\section{Estrategias 0 acciones implementadas para hacer frente a la revolución digital y al crecimiento de las redes sociales}

Respecto a la necesidad de gestionar los retos que ha traído consigo la revolución digital y el crecimiento de los social media, los líderes españoles aplican en su trabajo diario una serie de estrategias y acciones que pretenden minimizar los riesgos y aprovechar las oportunidades que se presentan. Los profesionales españoles afirman que, principalmente, están revisando las estrategias de comunicación con el objetivo de incorporar un mayor uso de los medios sociales y también monitorizan la comunicación de los públicos en la web social. Estos datos ofrecen más evidencias sobre los profundos cambios que han llegado de la mano de estas nuevas formas de comunicación. Además, para afrontar este reto también consideran importante entrenar a los miembros del equipo y el resto de empleados en el uso y las estrategias de medios sociales.

\section{Estrategias 0 acciones implementadas para lidiar con la velocidad y el volumen del flujo de información}

Usar nuevas tecnologías empleadas para recoger, analizar y distribuir más rápido las noticias y la información disponible $(M=5,81, S D=1,239)$, además de desarrollar nuevas habilidades y mejorar los procesos de trabajo $(\mathrm{M}=5,42, \mathrm{SD}=1,484)$, son las dos estrategias o acciones más implementadas para lidiar con la velocidad y el volumen del flujo de información. Los resultados obtenidos muestran que los profesionales todavía no han averiguado cuál es la mejor forma de manejar este problema o que las aproximaciones iniciales no han dado los resultados esperados. Un aspecto 


\section{Tabla 4}

\begin{tabular}{|l|c|c|}
\hline \multicolumn{1}{|c|}{ La revolución digital y el crecimiento de los social media } & M & SD \\
\hline Contratar empleados con habilidades especializadas en medios digitales & 5,13 & 1,714 \\
\hline Entrenar a los miembros del equipo o a otros empleados en el uso y estrategias de medios sociales & 5,62 & 1,600 \\
\hline Crear indicadores clave de actuación para medir las actividades en medios sociales & 5,18 & 1,614 \\
\hline Monitorizar la comunicación de los públicos en la web social & 5,78 & 1,506 \\
\hline Revisar las estrategias de comunicación para incorporar un mayor uso de los medios sociales & 5,96 & 1,537 \\
\hline
\end{tabular}

Nota: Escala Likert de 7 puntos entre 'poco importante' (1) y 'muy importante' (7).

relevante es que las organizaciones no están contratando nuevos emplea$\operatorname{dos}(\mathrm{M}=2,23, \mathrm{SD}=1,428)$ o incrementado el uso de consultores externos o agencias $(M=2,88, S D=1,776)$ para afrontar este problema. Sin duda, esta falta de iniciativa en las nuevas contrataciones está también ligada a la situación de recesión económica y sus consecuencias en la pérdida de recursos por parte de los departamentos de comunicación.

\section{Tabla 5}

\begin{tabular}{|l|c|c|}
\hline \multicolumn{1}{|c|}{ Lidiar con la velocidad y el volumen del flujo de información } & M & SD \\
\hline Usar nuevas tecnologías para recoger, analizar y distribuir más rápido noticias e información & 5,81 & 1,239 \\
\hline Desarrollar nuevas habilidades o mejorar los procesos de trabajo en su unidad & 5,42 & 1,484 \\
\hline Asignar trabajo adicional y responsabilidades a empleados que ya están en la unidad & 4,30 & 1,884 \\
\hline Incrementar el uso de consultores externos o agencias & 2,88 & 1,776 \\
\hline Contratar empleados adicionales permanentes o a media jornada & 2,23 & 1,428 \\
\hline
\end{tabular}

Nota: Escala Likert de 7 puntos entre 'poco importante' (1) y 'muy importante' (7).

\section{Estrategias 0 acciones implementadas con el objetivo de mejorar la medición de la eficacia de la comunicación para demostrar valor}

Seguir y analizar la cobertura de la organización y sus competidores o clientes en los medios de comunicación $(\mathrm{M}=5,27, \mathrm{SD}=1.756)$ es claramente la acción más empleada a la hora de mejorar la medición de la eficacia de la comunicación con el objetivo de demostrar el valor de las relaciones públicas. Así pues, los líderes de comunicación en España siguen apostando por métodos de medición de impacto frente a la medición de diversos niveles (Cutlip, Center y Broom, 2000; DPRG/ECV, 2009; Macnamara; 1999, 2001, 2002; Xavier, Mehta y Gregory, 2007) en lugar de avanzar hacia mo- 
delos más centrados en el valor de su trabajo en coordinación con las estrategias y objetivos corporativos. Como se aprecia en la tabla 7, la evaluación de las estrategias o las acciones implementadas es uno de los asuntos que sigue sin resolverse adecuadamente por parte de las organizaciones españolas. A excepción del análisis de la cobertura de los medios de comunicación y de la utilización de métricas económicas, el resto de las medidas son escasamente utilizadas. En los últimos años, se ha popularizado el ROI ( $r e-$ turn on investment) como sistema de evaluación, aunque otros estudios han despertado dudas acerca de a su correcta utilización (Zerfass et al. 2011).

\section{Tabla 6}

\begin{tabular}{|l|c|c|}
\hline \multicolumn{1}{|c|}{ Mejorar la medición de la eficacia de la comunicación para demostrar valor } & M & SD \\
\hline Usar métricas económicas para calcular la efectividad a nivel de rendimiento & 4,58 & 2,101 \\
\hline Seguir y analizar la cobertura de medios de la organización y sus competidores o clientes & 5,27 & 1,756 \\
\hline $\begin{array}{l}\text { Contratar expertos externos con conocimientos o habilidades de medición. y desarrollo de } \\
\text { métricas }\end{array}$ & 3,81 & 2,154 \\
\hline Asistir a talleres sobre medición para aprender a adoptar las mejores prácticas & 3,96 & 2,144 \\
\hline Centarse más en los indicadores de rendimiento no financieros que en las métricas financieras & 4,04 & 1,865 \\
\hline
\end{tabular}

Nota: Escala Likert de 7 puntos entre 'poco importante' (1) y 'muy importante' (7).

\section{Estrategias 0 acciones implementadas para afrontar con eficacia las posibles crisis}

Desarrollar planes efectivos de comunicación de crisis $(\mathrm{M}=5,08, \mathrm{SD}=1,875)$ es la estrategia más utilizada para afrontar con éxito los imprevistos que pueden surgir en las organizaciones, junto con la implementación de programas efectivos de gestión de asuntos estratégicos para reducir los riesgos de crisis $(M=4,92, S D=1,671)$. Se aprecia, sin embargo, una alta valoración de todas las acciones propuestas, aunque existe un mayor acuerdo sobre las medidas de acción que sobre las de escucha.

\section{Estrategias 0 acciones implementadas para afrontar la creciente demanda de transparencia en comunicaciones y operaciones}

Los líderes españoles en relaciones públicas parecen estar dispuestos a poner en práctica los principios dialógicos de la comunicación y apuestan por la transparencia. A pesar de que los resultados del estudio muestran un amplio uso de las cinco estrategias o acciones propuestas, los encuestados se decantan por la implementación de una estrategia general que permita au- 


\section{Tabla 7}

\begin{tabular}{|l|c|c|}
\hline \multicolumn{1}{|c|}{ Estar preparados para afrontar con eficacia las posibles crisis } & M & SD \\
\hline Desarrollar planes efectivos de comunicación de crisis & 5,08 & 1,875 \\
\hline $\begin{array}{l}\text { Utilizar la exploración de temas de interés y las tecnologías de seguimiento para identificar y } \\
\text { rastrear problemas potenciales }\end{array}$ & 4,42 & 1,579 \\
\hline Entrenar a los empleados en procedimientos de gestión de crisis & 4,81 & 1,650 \\
\hline $\begin{array}{l}\text { Educar a los stakeholders sobre comunicación de emergencia y sus correspondientes sistemas de } \\
\text { respuesta }\end{array}$ & 4,69 & 1,594 \\
\hline Implementar programas efectivos de gestión de asuntos de interés para reducir los riesgos de crisis & 4,92 & 1,671 \\
\hline
\end{tabular}

Nota: Escala Likert de 7 puntos entre 'poco importante' (1) y 'muy importante' (7).

mentar la transparencia en toda la organización $(\mathrm{M}=5.67, \mathrm{SD}=1.579)$. También existe un elevado consenso respecto a la necesidad de publicar más información sobre la compañía en Internet o Intranet y de monitorizar la comunicación con los diferentes públicos para identificar una posible preocupación por la falta de transparencia. Aunque con una alta puntuación, la estrategia menos apoyada sigue siendo la de ofrecer más oportunidades de comunicación bidireccional. La comunicación dialógica que forma parte de la esencia de los nuevos medios supone una inevitable pérdida de control de la organización como emisora, con unos resultados aún inciertos y, en muchas ocasiones, difícil de conjugar con la cultura de organizaciones y primeros ejecutivos españoles.

\section{Tabla 8}

\begin{tabular}{|l|c|c|}
\hline \multicolumn{1}{|c|}{ Afrontar la creciente demanda de transparencia en comunicaciones y operaciones } & M & SD \\
\hline Publicar más información sobre la compañía en Internet o Intranet & 5,58 & 1,283 \\
\hline $\begin{array}{l}\text { Seguimiento de la comunicación con los públicos para identificar una posible preocupación } \\
\text { por la transparencia }\end{array}$ & 5,50 & 1,351 \\
\hline Comunicar directamente con grupos externos para abordar asuntos de transparencia & 5,46 & 1,532 \\
\hline Ofrecer más oportunidades para la comunicación bidireccional entre empleados ylíderes & 5,25 & 1,726 \\
\hline $\begin{array}{l}\text { Implementar una estrategia general para incrementar la transparencia en toda la } \\
\text { organización }\end{array}$ & 5,67 & 1,579 \\
\hline
\end{tabular}

Nota: Escala Likert de 7 puntos entre 'poco importante' (1) y 'muy importante' (7).

\section{Estrategias 0 acciones implementadas para buscar, desarrollar y conservar a los profesionales de comunicación con gran talento}

Los encuestados que señalaron como problema la búsqueda, el desarrollo y la retención de los profesionales de comunicación con gran talento se mos- 
traron partidarios de conceder una mayor autonomía en el trabajo a los individuos que muestan un talento superior $(\mathrm{M}=5,17, \mathrm{SD}=2,082)$, además de apoyar la educación en las universidades y diseñar planes de desarrollo individualizados para los profesionales de alto potencial. Por el contrario, las estrategias encaminadas a proporcionar incentivos financieros y beneficios superiores a las personas talentosas o de recurrir a agencias de empleo para ayudar a localizar y evaluar el talento fueron menos valoradas por los encuestados. El alto nivel de desempleo en España, una circunstancia que también afecta al sector de las relaciones públicas y la gestión de la comunicación, puede explicar esta tendencia, ya que existe una gran oferta de trabajadores altamente cualificados a disposición de las organizaciones.

Tabla 9

\begin{tabular}{|l|c|c|}
\hline Buscar, desarrollar y conservar a los profesionales de comunicación con gran talento & M & SD \\
\hline Usar agencias de empleo para ayudar a localizar y evaluar el talento & 2,58 & 1,832 \\
\hline Proporcionar incentivos financieros y beneficios superiores al mayor talento & 3,33 & 2,270 \\
\hline Apoyar la educación de los futuros profesionales en las universidades & 4,33 & 2,348 \\
\hline Diseñar planes de desarrollo individualizado para profesionales de alto potencial & 4,33 & 2,462 \\
\hline Proporcionar una mayor autonomía en el trabajo a los individuos con más talento & 5,17 & 2,082 \\
\hline
\end{tabular}

Nota: Escala Likert de 7 puntos entre 'poco importante' (1) y 'muy importante' (7).

\section{Estrategias 0 acciones implementadas para afrontar las necesidades de comunicación en culturas diversas y mercados globalizados}

La internacionalización se ha convertido en un requisito que deben cumplir los líderes de relaciones públicas y que, a juzgar por los resultados de la investigación, es asumido de forma mayoritaria por los profesionales españoles. Seguir y analizar la cobertura de los medios y desarrollos en mercados globales es la estrategia preferida para enfrentar las necesidades de comunicación en culturas diversas y mercados globalizados $(M=5,64, S D=1,286)$, junto con el hecho de implementar estrategias de comunicación global para la organización $(\mathrm{M}=5,55, \mathrm{SD}=1,640)$.

\section{Desarrollo de futuros líderes}

Los profesionales son muy conscientes de las consecuencias de la globalización. No en vano, cuando proyectan las necesidades de los líderes del futuro, el cambio y la escucha se sitúan en cabeza. Respecto a las acciones que 


\section{Tabla 10}

\begin{tabular}{|l|c|c|}
\hline \multicolumn{1}{|c|}{ Enfrentar las necesidades de comunicación en culturas diversas y mercados globalizados } & M & SD \\
\hline Contratar más empleados con experiencia internacional o conocimientos de idiomas & 4,36 & 1,859 \\
\hline Proporcionar programas de entrenamiento cultural para empleados & 4,91 & 1,375 \\
\hline $\begin{array}{l}\text { Usar expertos nacionales para conducir los programas de comunicación en cada país de } \\
\text { operación }\end{array}$ & 4,91 & 1,640 \\
\hline Seguir y analizar la cobertura de los medios y desarrollos en mercados globales & 5,64 & 1,286 \\
\hline Implementar estrategias de comunicación global para la organización & 5,55 & 1,368 \\
\hline
\end{tabular}

Nota: Escala Likert de 7 puntos entre 'poco importante' (1) y 'muy importante' (7).

pueden contribuir al desarrollo de los futuros líderes de comunicación y al fortalecimiento de la profesión, los encuestados ponen el acento en la formación y consideran especialmente positivo el fortalecimiento de las habilidades y capacidades de gestión del cambio $(\mathrm{M}=5,77, \mathrm{SD}=1,205)$, además de la mejora de las habilidades de escucha $(M=5,67, S D=1,077)$ y las relacionadas con la gestión de conflictos $(\mathrm{M}=5,51, \mathrm{SD}=1,138)$.

La necesidad de responder a los desafíos de las nuevas formas de comunicación digital al alcance de los stakeholders y a los cambios experimentados en la profesión parece estar detrás de estas respuestas. A continuación aparecen medidas relacionadas con el reto de incrementar la comprensión y la sensibilidad cultural $(M=5,35, S D=1,346)$, imponer sanciones duras a los que violen las normas éticas $(\mathrm{M}=5,33, \mathrm{SD}=1,461)$ y desarrollar medidas, tanto para demostrar el valor de las relaciones públicas, como para mejorar la inteligencia emocional de los profesionales. Un aspecto a destacar es que en la posiblidad de sancionar duramente a los que violen las normas éticas existe una brecha intergeneracional: los encuestados con más de 20 años de experiencia se muestran más partidarios de esta medida $(M=5,53$, $\mathrm{SD}=1,386)$ que los más inexpertos.

Por otra parte, los profesionales españoles no consideran importante instar a las asociaciones profesionales a trabajar conjuntamente para desarrollar líderes $(M=4,81, S D=1,254)$ ni requerir acreditaciones o licencias profesionales $(M=4,60, S D=1,621)$. Estas afirmaciones coinciden con el conocimiento aportado por estudios previos (Moreno et al., 2010, 2011) 
en los que ya se detectaba que los profesionales españoles rechazan mayoritariamente la normativización del acceso a la profesión.

A pesar de que la mejora de la evaluación aparecía como el tercer reto que más preocupaba a los líderes de las relaciones púbicas y gestión de la comunicación, no recibió la misma valoración cuando se planteó el fututo de la profesión. Desarrollar medidas más eficaces para documentar el valor y la contribución de las relaciones públicas se sitúa en la sexta posición entre la acciones recomendadas para la próxima generación de líderes $(M=5,30, S D=1,348)$.

El análisis de los datos muestra que los profesionales jóvenes consideran más importante mejorar las habilidades profesionales para gestionar el estrés laboral $(M=5,44, S D=1,162)$ que los más experimentados $(M=4,72$, $\mathrm{SD}=1,792$ ). Por otra parte, la necesidad de mejorar las habilidades de escucha de los profesionales es más valorada por los encuestados que trabajan en agencias de comunicación $(\mathrm{M}=6,02, \mathrm{SD}=0,911)$ y organizaciones sin ánimo de lucro $(\mathrm{M}=5,71, \mathrm{SD}=1,157)$ que entre los responsables de comunicación de empresas privadas $(\mathrm{M}=5,30, \mathrm{SD}=1,153)$.

\section{Tabla 11}

\begin{tabular}{|l|c|c|}
\hline \multicolumn{1}{|c|}{ Acciones recomendadas para mejorar la profesión y el desarrollo de los futuros líderes } & M & SD \\
\hline Fortalecer las habilidades y capacidades de gestión del cambio & 5,77 & 1,205 \\
\hline Mejorar las habilidades de escucha de los profesionales & 5,67 & 1,077 \\
\hline Mejorar las habilidades de gestión de conflictos & 5,51 & 1,138 \\
\hline Incrementar la comprensión y sensibilidad cultural & 5,35 & 1,346 \\
\hline Imponer sanciones duras a los que violen las normas éticas & 5,33 & 1,461 \\
\hline $\begin{array}{l}\text { Desarrollar medidas más eficaces para documentar el valor y la contribución de las relaciones } \\
\text { públicas }\end{array}$ & 5,30 & 1,348 \\
\hline $\begin{array}{l}\text { Desarrollar entrenamientos para mejorar la inteligencia emocional de los profesionales de } \\
\text { relaciones públicas }\end{array}$ & 5,29 & 1,256 \\
\hline $\begin{array}{l}\text { Fortalecer el componente de negocio/económico de los programas de educación en } \\
\text { comunicación }\end{array}$ & 5,09 & 1,332 \\
\hline Desarrollar un plan de estudios básico global de educación & 4,95 & 1,452 \\
\hline Mejorar las habilidades profesionales para gestionar el estrés laboral & 4,95 & 1,439 \\
\hline Instar a las asociaciones profesionales a trabajar conjuntamente para desarrollar líderes & 4,81 & 1,254 \\
\hline Requerir acreditaciones o licencias profesionales & 4,60 & 1,621 \\
\hline
\end{tabular}

Nota: Escala Likert de 7 puntos entre 'poco importante' (1) y 'muy importante' (7). 
Del análisis factorial de componentes principales con los 12 ítems relacionados con las acciones necesarias para la mejora del liderazgo han resultado tres factores (tabla 15) que explican el 64,2\% de la varianza explicada $(\mathrm{KMO}=0,877, \mathrm{p} \leq 000)$. El primer factor $(26,3 \%$ de la varianza explicada) hace referencia a aquellas acciones encaminadas a mejorar la gestión del trabajo dentro de la propia organización en la que se ejerce el liderazgo. El segundo grupo de ítems (19,6\% de la varianza explicada) encuadra las acciones relacionadas con la regulación de la profesión en los ámbitos formativo y deontológico. Por último, el factor tres (18,3\% de la varianza explicada) revela la necesidad de promover la proyección externa de la profesión tanto a través de las asociaciones como las estrategias que pongan en valor la contribución de las relaciones públicas en las organizaciones.

\section{Tabla 12}

\begin{tabular}{|c|c|c|c|c|}
\hline & $\mathbf{M}$ & $\begin{array}{c}\text { Acciones } \\
\text { profesionales }\end{array}$ & $\begin{array}{l}\text { Regulación } \\
\text { profesional }\end{array}$ & $\begin{array}{c}\text { Proyección } \\
\text { externa }\end{array}$ \\
\hline Mejorar las habilidades de escucha de los profesionales & 5,67 & 0,811 & & \\
\hline Incrementar la comprensión y la sensibilidad cultural & 5,35 & 0,788 & & \\
\hline $\begin{array}{l}\text { Desarrollar entrenamientos para mejorar la inteligencia emocional } \\
\text { de los profesionales de relaciones públicas }\end{array}$ & 5,29 & 0,718 & & \\
\hline Fortalecer las habilidades y capacidades de gestión del cambio & 5,09 & 0,686 & & \\
\hline Mejorar las habilidades profesionales para gestionar el estrés laboral & 5,51 & 0,572 & & \\
\hline Mejorar las habilidades de gestión de conflictos & 5,51 & 0,536 & & \\
\hline Imponer sanciones duras a los que violen las normas éticas & 5,33 & & 0,827 & \\
\hline Desarrollar un plan de estudios básico global de educación & 4,95 & & 0,788 & \\
\hline Requerir acreditaciones o licencias profesionales & 4,60 & & 0,682 & \\
\hline $\begin{array}{l}\text { Desarrollar medidas más eficaces para documentar el valor y la } \\
\text { contribución de las relaciones públicas }\end{array}$ & 5,30 & & & 0,800 \\
\hline $\begin{array}{l}\text { Fortalecer el componente de negocio/económico de los programas } \\
\text { de educación en comunicación }\end{array}$ & 5,77 & & & 0,750 \\
\hline $\begin{array}{l}\text { Instar a las asociaciones profesionales a trabajar conjuntamente } \\
\text { para desarrollar líderes }\end{array}$ & 4,81 & & & 0,579 \\
\hline
\end{tabular}

Los factores se convirtieron en tres nuevas variables con el objetivo de encontrar posibles diferencias de medias en función de las características sociodemográficas y profesionales de los encuestados. Sólo se encontraron estas diferencias en el primer factor cuando se comparó con el nivel de estudios $(\mathrm{F}=7,394, \mathrm{p} \leq 000)$ : quienes no han realizado estudios universitarios valoran en menor medida los ítems de este grupo. También en este 
factor las diferencias de medias son estadísticamente significativas en la variable estudios realizados $(\mathrm{F}=2,769, \mathrm{p} \leq 003)$. Destaca que quienes han realizado estudios de relaciones públicas valoran en menor medida los ítems de este factor.

\section{Creencias personales y percepciones sobre el liderazgo}

\section{Autopercepción como líder}

Respecto a la autopercepción como líder en relaciones públicas y gestión de la comunicación, los profesionales se muestran de acuerdo sobre las ventajas de aprender a través de modelos de rol o de tutores dentro del trabajo, por encima de la educación universitaria o de los programas de desarrollo gerencial $(\mathrm{M}=4,90, \mathrm{SD}=1,613)$. Además, la mayoría se considera a sí mismo como un líder en el sector $(\mathrm{M}=4,78, \mathrm{SD}=1,468)$ y un pequeño porcentaje afirma que no quiere serlo.

\section{Tabla 13}

\begin{tabular}{|l|c|c|}
\hline \multicolumn{1}{|c|}{ Autopercepción como líder } & M & SD \\
\hline Me considero un líder en relaciones públicas, & 4,78 & 1,468 \\
\hline No quiero ser un líder en relaciones públicas, & 2,45 & 1,693 \\
\hline $\begin{array}{l}\text { Aprendo más sobre la excelencia del liderazgo a través de los modelos de rol o tutores en el } \\
\text { trabajo, que desde la educación universitaria o programas de desarrollo gerencial }\end{array}$ & 4,90 & 1,613 \\
\hline
\end{tabular}

Nota: Escala Likert de 7 puntos entre 'poco importante' (1) y 'muy importante' (7).

\section{Género y liderazgo}

Existe un consenso general sobre la igualdad de género en el ámbito del liderazgo en relaciones públicas $(\mathrm{M}=6,73, \mathrm{SD}=0,837)$, aunque todavía encontramos algunos profesionales que prefieren a un hombre como líder $(\mathrm{M}=2,29, \mathrm{SD}=1,746)$. Respecto a la teóricamente aceptada 'feminización' de la profesión, un importante número de encuestados en España cree que las mujeres no poseen mejores habilidades de comunicación interpersonal que los hombres.

\section{Condiciones organizacionales}

Los datos nos muestran un resultado sorprendente y es que los profesionales valoran más el papel del primer ejecutivo respecto a la comunicación que 


\section{Tabla 14}

\begin{tabular}{|c|c|c|}
\hline Género y liderazgo & M & SD \\
\hline Hombres y mujeres pueden ser líderes igualmente capaces en relaciones públicas & 6,73 & 0,837 \\
\hline Prefiero trabajar para un líder masculino en el trabajo & 2,29 & 1,746 \\
\hline Las mujeres no tienen mejores habilidades de comunicación interpersonal que los hombres & 4,53 & 1,997 \\
\hline
\end{tabular}

Nota: Escala Likert de 7 puntos entre 'poco importante' (1) y 'muy importante' (7).

el del CCO o director de comunicación. Como se observa en la tabla 19, los encuestados no evaluaron de forma muy positiva el comportamiento del líder de comunicación de su organización, a pesar de que un número importante se había definido previamente como líder $(\mathrm{M}=4,71, \mathrm{SD}=1,879)$. De la misma manera, la afirmación sobre la práctica de la comunicación bidireccional entre los miembros de la organización obtuvo resultados similares, mostrando que existe todavía un largo camino que recorrer hacia esta práctica de la excelencia. Sin embargo, los profesionales piensan que el CEO o el ejecutivo en una posición más elevada sí entiende el valor de las relaciones públicas $(\mathrm{M}=5,24, \mathrm{SD}=1,981)$. La percepción organizacional es más positiva en agencias y entidades públicas que en organizaciones sin ánimo de lucro o empresas privadas.

\section{Tabla 15}

\begin{tabular}{|l|c|c|}
\hline \multicolumn{1}{|c|}{ Condiciones organizacionales } & M & SD \\
\hline El profesional en el nivel más alto de mi organización es un líder excelente & 4,71 & 1,879 \\
\hline Mi organización alienta y practica la comunicación bidireccional entre sus miembros & 4,45 & 1,915 \\
\hline El CEO o el ejecutivo más alto de mi organización entiende el valor de las relaciones públicas & 5,24 & 1,981 \\
\hline
\end{tabular}

Nota: Escala Likert de 7 puntos entre 'poco importante' (1) y 'muy importante' (7).

\section{Impacto en la profesión}

Los encuestados muestran un cauteloso optimismo sobre el futuro de la profesión de relaciones públicas en España $(M=4,75, \mathrm{SD}=1,678)$ y un escaso acuerdo respecto a la mayor importancia de las habilidades de liderazgo sobre las habilidades de comunicación en la dirección o el departamento de relaciones públicas $(M=3,31, S D=1,680)$. Los profesionales españoles no creen que existan diferencias entre el liderazgo en la gestión de la comunicación y en otras disciplinas $(\mathrm{M}=3,99, \mathrm{SD}=2,035)$. 


\section{Tabla 16}

\begin{tabular}{|l|c|c|}
\hline \multicolumn{1}{|c|}{ Impacto en la profesión } & M & SD \\
\hline El liderazgo en la gestión de comunicación es diferente del liderazgo en otros ámbitos & 3,99 & 2,035 \\
\hline $\begin{array}{l}\text { Las habilidades de liderazgo son más importantes que las habilidades de comunicación en } \\
\text { la dirección o el departamento de relaciones públicas }\end{array}$ & 3,31 & 1,680 \\
\hline Soy optimista sobre el futuro de la profesión de relaciones públicas en mi país & 4,75 & 1,678 \\
\hline
\end{tabular}

Nota: Escala Likert de 7 puntos entre 'poco importante' (1) y 'muy importante' (7).

\section{Discusión y conclusiones}

El estudio del liderazgo en el campo de las relaciones públicas en España no ha recibido la atención y el interés que merece por su importancia estratégica en la gestión de la comunicación dentro de las organizaciones. Esta investigación exploratoria ha tratado de profundizar en las habilidades, actitudes y comportamientos que promueven un liderazgo excelente y, al mismo tiempo, ha identificado los retos más importantes a los que se enfrentan los profesionales responsables de la función de comunicación.

Respecto a los asuntos de interés considerados como más importantes para el desempeño diario del liderazgo en relaciones públicas, los dos más destacados tienen que ver los con social media y sus efectos: gestionar la revolución digital y lidiar con la velocidad y el volumen del flujo de información. Estos resultados son consistentes con los escasos estudios previos sobre la situación actual de la profesión en España, en los que se detecta una creciente preocupación sobre los desafíos que han llegado de la mano de la rápida y masiva introducción de los medios digitales en la comunicación organizacional. La dificultad de definir y localizar los públicos clave, monitorizar las conversaciones que se producen en las redes sociales y responder de forma rápida y apropiada a las necesidades informativas que plantean son algunos de los retos que deben afrontar los líderes actuales. El futuro de las organizaciones dependerá en gran medida de cómo sean capaces de gestionar $y$ resolver estas cuestiones.

Mejorar la evaluación de la comunicación para poder demostrar su valor es otra de las preocupaciones de los profesionales del sector. Sin embargo, la mayoría de los encuestados declara seguir limitándose al estadio 
evaluativo del impacto, y en concreto de las comunicaciones mediáticas. Este estudio también pone de manifiesto que existe una conciencia cada vez más profunda sobre la necesidad de estar preparado para afrontar las crisis actuales y las que puedan ocurrir en el futuro.

Respecto a las condiciones, habilidades y aptitudes que los líderes necesitan para afrontar los principales retos que encuentran en su trabajo, los profesionales españoles destacan la importancia de participar en la toma de decisiones estratégicas dentro de la organización. También valoran los conocimientos sobre comunicación para poder desarrollar estrategias, planes y mensajes apropiados, además de la capacidad de ofrecer una visión convincente de cómo la comunicación puede ayudar a la organización.

Para hacer frente a la revolución digital, la principal acción implementada consiste en revisar las estrategias de comunicación para incorporar un mayor uso de los medios sociales, mientras que usar nuevas tecnologías para recoger, analizar y distribuir más rápido noticias e información es la acción más adecuada para lidiar con la velocidad y el volumen del flujo de información.

Sobre las acciones que pueden contribuir al desarrollo de los futuros líderes en este campo y al fortalecimiento de la profesión, la formación se sitúa en un puesto destacado. Los profesionales se decantan sobre todo por mejorar las habilidades de gestión del cambio, de escucha y de gestión de conflictos. Incrementar la comprensión y la sensibilidad cultural, imponer sanciones duras a los que violen las normas éticas y desarrollar medidas más eficaces para documentar el valor y la contribución de las relaciones públicas son otras de las propuestas más valoradas.

Por último, en cuanto a la autopercepción como líderes en relaciones públicas y gestión de la comunicación se observa un alto nivel de acuerdo respecto a la capacidad de hombres y mujeres para ejercer el liderazgo. Aunque en menor medida, también existe consenso sobre la posibilidad de aprender más de los líderes excelentes en el trabajo que de los programas educativos universitarios. En este aspecto, no se encuentran diferencias estadísticamente significativas entre hombres y mujeres. 
Este estudio es un primer intento de analizar la dinámica del liderazgo en el campo de las relaciones públicas y la gestión de la comunicación en España, un esfuerzo motivado por el deseo de profundizar en este complejo y polifacético concepto. La ausencia de investigaciones empíricas previas es un obstáculo a la hora de establecer comparaciones o analizar la evolución del liderazgo a lo largo del tiempo. A pesar de que esta investigación se basa en un análisis empírico sobre la población objeto de estudio, el cuestionario deberá ser probado de nuevo en diferentes muestras a lo largo del tiempo. Algunos aspectos, como los asuntos más importantes para los líderes de comunicación, son claramente circunstanciales y habrán de ser actualizados y adaptarse a las circunstancias y retos del futuro.

Esta investigación desarrolla un modelo de estudio que ofrece la posibilidad de conocer más en profundidad la complejidad de este fenómeno y los factores clave para la práctica de un liderazgo excelente en las organizaciones. Posteriores análisis permitirán comparar estas conclusiones del sector en España con otros países y validar el instrumento en diversos contextos culturales, políticos y económicos.

La dificultad de conceptualizar y medir frena el progreso hacia la comprensión de este importante objeto de estudio. Existe una gran variedad de definiciones y para entender sus efectos en el comportamiento organizacional es necesario examinar múltiples niveles del liderazgo ejecutivo de forma simultánea. En este sentido, este artículo ofrece un encuadre conceptual básico que puede resultar de utilidad para el estudio del liderazgo en el contexto de las relaciones públicas, con implicaciones tanto a nivel académico como profesional.

\section{Referencias}

Adedec (2002). La comunicación y las relaciones públicas en España. Radiografía de un sector. Madrid: Pirámide.

Adedec (2004). La comunicación y las relaciones públicas en España. Radiografía de un sector. Madrid. Disponible en: http://www.adecec.com [fecha de consulta: 7 de enero de 2012]. 
Adedec (2008). La comunicación y las relaciones públicas en España. Radiografía de un sector. Madrid. Disponible en: http://www.adecec. com [fecha de consulta: 7 de enero de 2012].

Aldoory, L. y Toth, E. (2004). "Leadership and gender in public relations: Perceived effectiveness of transformational and transactional leadership styles". En: Journal of Public Relations Research, 16 (2), pp. 157-183.

Berger, B. K. (2005). "Power Over, Power With, and Power to Relations: Critical Reflections on Public Relations, the Dominant Coalition, and Activism”. En: Journal of Public Relations Research, 17 (1), pp. 5-28.

Berger, B. K. (2007). "Public Relations and Organizational Power”. En: Toth, E. L. (ed.). The Future of Excellence in PR and Communication Management (pp. 221-234). Mahwah, NJ: Lawrence Erlbaum.

Berger, B. K. y Meng, J. (2010). "Public Relations Practitioners and the Leadership Challenge”. En: Heath, R. L. (ed.). The Sage Handbook of Public Relations (pp. 421-434). Thousand Oaks, CA: Sage Publications.

Berger, B. K. y Reber, B. H. (2006). Gaining Influence in Public Relations: The Role of Resistance in Practice. Mahwah, NJ: Lawrence Erlbaum.

Berger, B. K., Reber, B. H. y Heyman, W. C. (2007). "You can't homogenize success in communication management: PR leaders take diverse paths to top". En: International Journal of Strategic Communication, 1 (1), pp. 53-71.

Choi, Y. y Choi, J. (2009). "Behavioral dimensions of public relations leadership in organizations”. En: Journal of Communication Management, 13, pp. 292-309.

Costa, J. (2009). El dircom hoy. Dirección y gestión de la comunicación en la nueva economía. Barcelona: Costa Punto Com (2a edición 2010). 
Costa, J. (2011). El ADN del dircom. Origen, necesidad, expansión y futuro de la dirección de comunicación. Barcelona: Costa Punto Com.

Cutlip, S. M., Center, A. H. y Broom, G. M. (2000). Effective Public Relations. Nueva Jersey: Prentice-hall.

Dircom (2000). El estado de la comunicación en España: empresas y organismos públicos e instituciones. Madrid: Dircom.

Dircom (2005). El estado de la Comunicación en España. Madrid: Dircom.

Dircom (2010). El estado de la Comunicación en España. Madrid: Dircom.

Grunig, J. E. (ed.) (1992). Excellence in Public Relations and Communication Management. Hillsdale, NJ: Lawrence Erlbaum.

Grunig, L. A., Grunig, J. E. y Dozier, D. M. (2002). Excellent Public Relations and Effective Organizations. Mahwah, NJ: Lawrence Erlbaum.

Horner-Long, P. y Schoenberg, R. (2002). "Does e-Business Require Different Leadership Characteristics? An empirical investigation”. En: European Management Journal, 20 (6), pp. 611-619.

Gutiérrez-García, E. (2010). "Gobierno corporativo y comunicación empresarial. ¿Qué papel cumplen los directores de comunicación en España?". En: Palabra Clave, 3 (1), pp. 147-160.

Macnamara, J. (1999). "Research in public relations: A review of the use of evaluation and formative research". En: Asia Pacific Public Relations Journal, 1 (2), pp. 107-133.

Macnamara, J. (2001). Professional bodies condemn AVEs. Disponible en: www.pria.com.au [fecha de consulta: 22 de diciembre de 2012].

Macnamara, J. (2002). “Research and Evaluation”. En: Tymson, C., Lazar, P. y Lazar, R. (eds.). The New Australian and New Zealand Public Relations Manual (pp. 100-135). Sydney: Tymson Communications. 
Matilla, K. (2010). “Pasado, presente y futuro del 'dircom' en España. En: Fisec-Estrategias. Facultad de Ciencias Sociales de la Universidad Nacional de Lomas de Zamora, año V, 14 (4), pp. 3-24.

Matilla, K. y Marca, G. (2011). "La función estratégica del dircom en España en 2010”. En: Revista Internacional de Relaciones Públicas, 2 (1), pp. 11-23.

Meng, J. (2009). "Excellent leadership in public relations: An application of multiple-group confirmatory factor analysis models in assessing cross-national measurement in variance”. Trabajo inédito. Tuscaloosa, AL: University of Alabama.

Meng, J. y Berger, B. K. (2010). "Cultural influence on the perceptions of effective leadership in public relations". Ensayo presentado en la 60 Convención Anual de la Asociación Internacional de Comunicación. Singapur.

Meng, J. y Berger, B. K. (2011). "Strategic Leadership in Public Relations: Dimensions, Measurement, and Validation”. Ensayo presentado en la 13 Conferencia Anual Internacional de Investigación en Relaciones Públicas. Miami.

Meng, J. y Heyman, W. (2009). "Measuring excellent leadership in public relations: A second-order factor model in the dimension of selfdynamics". Ensayo presentado en la 12 Conferencia Internacional de Investigación en Relaciones Públicas. Miami.

Meng, J., Berger, B., Gower, K. y Heyman, W. (2012). "A test of excellent leadership in public relations: Key qualities, valuable sources, and distinctive leadership perceptions". En: Journal of Public Relations Research, 24 (1), pp. 18-36.

Morales, F. y Enrique, A. M. (2007). 2La figura del dircom. Su importancia en el modelo de comunicación integral”. En: Análisi, 35, pp. 83-93. 
Moreno, M. A. (2004). “Spain”. En: Van Ruler, B. y Verčič, D. (eds.). Public Relations and Communication Management in Europe (pp. 393412). Berlín: Mouton de Gruyter.

Moreno, Á., Zerfass, A. y Navarro, C. (2010). “La situación de los profesionales de relaciones públicas y comunicación estratégica en Europa y en España. Análisis comparado del European Communication Monitor 2009”. En: Actas Congreso AIPR. Barcelona.

Moreno, Á., Zerfass, A. y Navarro, C. (2011). "European Communication Monitor 2010: España en línea con los profesionales europeos”. En: VV. AA. Anuario de la Comunicación 2011. Madrid: Dircom.

Moreno, Á., Zerfass, A. y Navarro, C. (2012). “Tendencia de Comunicación en Europa. European Communication Monitor 2011”. En: VV.AA. Anuario de la Comunicación 2012. Madrid: Dircom.

Mut, M. (2006). "El Director de Comunicación, perfil de una nueva figura”. En: FISEC-Estrategias, Facultad de Ciencias Sociales de la Universidad Nacional de Lomas de Zamora, Año II, 5, pp. 3-23.

Pérez Beruete, C. y Sánchez Galindo, M. (2010). Nuevos modelos de gestión y función de los responsables de comunicación: estudio sobre el modelo español de gestión y reporting de intangibles para un Dircom. Madrid: EOI.

Smudde, P. M. y Courtright, J. L. (2010). “Public Relations and power”. En: Heath, R. L. (ed.). The Sage Handbook of Public Relations (pp. 177190). Thousand Oak, CA: Sage Publications.

Swerling, J., Sen, C., Bonefeste, A., Rezvan, A., Lee, D, y Mchargue, A. (2008). 10 Annnual Public Relations Generally Accepted Practices (G.A.P.) Study. GAP V. Los Ángeles: University of Southern California. Disponible en: http://annenberg.usc.edu/CentersandPrograms/ResearchCenters/SPRC.aspx [fecha de consulta: 2 de mayo de 2013]. 
Werder, K. P. y Holtzhausen, D. (2009). "An analysis of the influence of public relations department leadership style on public relations strategy use and effectiveness". En: Journal of Public Relations Research, 21 (4), pp. 404-427.

Xavier, R., Mehta, A. y Gregory, A. (2006). "Evaluation in use: The practitioner view of effective evaluation”. En: PRism, 4 (2): s.p.

Zerfass, A., Verhoeven, P., Tench, R., Moreno, A. y Vercic, D. (2011). European Communication Monitor 2011. Empirical Insights into Strategic Communication in Europe. Results of an Empirical Survey in 43 Countries. Bruselas: EACD, Euprera.

Zerfass, A., Vercic, D., Verhoeven, P., Moreno, A. y Tench, R. (2012). European Communication Monitor 2012. Challenges and Competencies for Strategic Communication. Results of an Empirical Survey in 42 Countries. Bruselas: EACD/Eeprera, Helios Media. 